
\title{
25 Research Square \\ QTL analysis based on a long-grain CSSL-Z1392 and SSSLs and fine mapping of qGL6
}

Fangming Zhao ( $\nabla$ zhaofangming2004@163.com )

Southwest University https://orcid.org/0000-0003-2781-0452

Ting Zhang

Southwest University

Shiming Wang

Southwest University

Shuangfei Sun

Southwest University

Yi Zhang

Southwest University

Juan Li

Southwest University

Jing You

Southwest University

Tian Su

Southwest University

Wenbo Chen

Southwest University

Yinghua Ling

Southwest University

Guanghua He

Southwest University

Original article

Keywords: Rice; chromosome fragment substitution line; grain length; QTL mapping; epistatic analysis

Posted Date: January 17th, 2020

DOI: https://doi.org/10.21203/rs.2.21138/v1

License: (9) (1) This work is licensed under a Creative Commons Attribution 4.0 International License.

Read Full License 


\section{Abstract}

Background: Grain shape affects not only rice yield but is also an important element in quality of appearance. However, the mechanism for inheritance of grain shape is unclear. Results: A rice chromosome segment substitution line Z1392, which harbors three substitution segments and produces grains of increased length, was identified. The three chromosome segments were located on chromosomes 1, 5, and 6, and the average length of the substitution segment was $4.29 \mathrm{Mb}$. Cytological analysis indicates that the predominant cause of increased grain length in Z1392 could be cell expansion in the glumes. 18 quantitative trait loci (QTLs) for important agronomic traits were identified. The inheritance of grain length in Z1392 was controlled by two major QTLs, qGL-5 and qGL-6. Finally, the qGL-6 was narrowed to a $1.26 \mathrm{Mb}$ region on chromosome 6, and OsARF19 may be the candidate gene of qGL-6. Based on QTL mapping, three single-segment substitution lines (S1, S2, and S3) and two doublesegment substitution lines (D1 and D2) were selected, and the mapping accuracy for qGL-5 and qGL-6 was further verified using the three single-segment substitution lines. Analysis of QTL additive and epistatic effects revealed that the additive effect of alleles qGL5 and qGL6 from 'Xihui 18' was estimated to increase grain length of Z1392 by 0.22 and $0.15 \mathrm{~mm}$, respectively. In addition, a positive epistatic interaction between qGL- 5 and qGL- 6 was detected, which indicates that the pyramiding of qGL-5 and qGL-6 for grain length produces a novel genotype with longer grains. Conclusions: Inheritance of grain length in the triple-segment substitution line $\mathrm{Z1392}$ is controlled by two major QTLs, qGL-5 and qGL-6 , qGL- 6 was narrowed to a $1.26 \mathrm{Mb}$ region on chromosome 6 , OsARF19 may be the candidate gene of qGL6 . And a positive epistatic interaction between qGL-5 and qGL-6 results in longer grains. The present results could be used to facilitate cloning of the qGL-5 and qGL-6 genes and contribute to improvement in grain yield in rice.

\section{Background}

Rice (Oryza sativa L.) is an important cereal crop throughout Asia. On the basis of the length: width ratio, rice grains are divided into three size classes, namely long, medium, and short grain, of which the ratios range from greater than 3.0, 2.1-2.9, and less than 2, respectively (Bai et al, 2010). Improvements in grain size are directly associated with yield, therefore grain size and yield-related traits are an important focus in research on rice. Chromosome fragment substitution lines (CSSLs) are invaluable material in quantitative trait locus (QTL) research for the separation and localization of specific traits (Kubo and Yoshimura, 2002). Quantitative traits, such as grain size, plant height, heading stage, and grain filling, are controlled by multiple genes. The construction of CSSLs has formed a strong foundation on which to base further research on quantitative traits.

Rice grain size is controlled by a combination of grain length, grain width, and grain thickness. Previous studies have shown that the development of grain size is dependent on multiple pathways. The mitogenactivated protein kinases (MAPK) pathway contains three cascade reactions, which play an important role in regulation of grain size (Li and Li, 2016). SMG1 encodes MITOGEN-ACTIVATED PROTEIN KINASE 4 (OsMAPK4), which is involved in the MAPK signaling pathway. The smg 1 mutant produces small and 
light grains due to a decreased cell number (Duan et al, 2014). OsMAPK6 may be a downstream effector of OSMAPK4. Mutation of OSMAPK6 also causes small-grain and dwarf phenotypes as a result of limited cell proliferation (Liu et al, 2015b). GRAIN SIZE AND NUMBER 1 (GSN1) is a negative regulator of the SMALL GRAIN 2 (OSMKKK10) -SMALL GRAIN 1 (OSMKK4) -OSMPK6 cascade reaction, and regulates cell differentiation and proliferation through the GSN1-MAPK pathway, thus regulating the number and length of grains (Guo et al, 2018). Guanine nucleotide-binding proteins ( $G$ proteins) consist of three subunits $(a, \beta$, and $Y)$, and $G$ protein-coupled receptors are also involved in the transduction of signaling pathways in rice (Liu et al, 2018). qLGY3 encodes a variable splicing protein, OsMADS1lgy3, which is a crucial effector downstream of the $\mathrm{G}$ protein $\beta \gamma$ dimer (Liu et al, 2018). The Gy subunit LONG KERNEL 3 (GS3) interacts with DENSE AND ERECT PANICLE 1 (DEP1) and MADS-domain transcription factors, and participates in the G-protein regulation pathway as a cofactor in the regulation of grain size. Concurrent expression of an allele of the grain size gene OSMADS1/gy3, GS3, and panicle DEP1 may result in traits that enhance yield and quality (Liu et al, 2018). Several recent studies have demonstrated that the ubiquitin-proteasome pathway may also regulate the development of grain size in rice. GRAIN WEIGHT 2 (GW2) encodes a RING-type protein with E3 ubiquitin ligase activity, which regulates proteolysis by targeting substrate binding to the proteasome, thereby negatively regulating cell proliferation and playing a role in the degradation of the ubiquitin-proteasome pathway. Loss of GW2 increases cell number, which results in broader glumes and an accelerated rate of grain filling, thus increasing grain width, weight, and yield (Song et al, 2007). Epigenetic modification also regulates the development of grain size. RELATED TO ABSCISIC ACID INSENSITIVE 3 (ABI3)/VIVIPAROUS 1 (VP1) 6 (RAV6) encodes a B3 DNAbinding protein, which affects the brassinosteroid (BR) pathway by controlling the degree of promoter methylation, thereby regulating the leaf angle and grain size (Zhang et al, 2015). GW6a encodes a novel GANT-like protein with histone acetyltransferase activity, which regulates rice grain size and yield by regulating the overall acetylation level of histone $\mathrm{H} 4$ (Song et al, 2015). In addition, many phytohormoneregulated pathways in rice control the proliferation and longitudinal growth of cells. Multiple genes are involved in the BR signaling pathway; for example, DWARF EBISU (D2/SMG11) encodes cytochrome P450, which regulates grain size by controlling cell elongation (Fang et al, 2016). Several genes are involved in auxin regulation, such as BIG GRAIN 1 (BG1), which encodes a novel membrane-localized protein. Overexpression of $B G 1$ leads to a significant increase in grain size, with clearly perturbed gravitropism in severe cases, by changing the auxin basipetal transport and altered auxin distribution; this suggests that $B G 1$ plays a role in the auxin regulatory pathway (Liu et al, 2015a). Genes involved in the cytokinin regulatory pathway, such as REGULATOR OF AWN ELONGATION 2 (GAD1), which encodes an epidermal pattern factor, regulate the content of endogenous cytokinins by interacting with GRAIN NUMBER 1A (OsCKX2) and DROUGHT AND SALT TOLERANCE (DST) to regulate grain size (Jin et al, 2016). In general, many types of regulatory modes of rice grain size are known, all of which require further study, owing to the complex regulatory modes within and among the pathways. Identification and cloning of novel genes are of considerable importance for studying the genes involved in the regulatory pathways of grain size, yield-related traits, and the interaction between genes, for selecting superior rice cultivars, and to improve grain yield and quality. 
In this study, a novel rice CSSL with long grains, Z1392, was identified. The CSSL was derived from a cross between 'Nipponbare' as the recipient parent and the indica restorer line 'Xihui 18' as the donor parent, and carried three substitution segments. We performed QTL mapping of important agronomical traits using a secondary $F_{2}$ population derived from the cross between 'Nipponbare' and Z1392. On the basis of the QTL mapping results, we selected the single-segment (SSSL), double-segment (DSSL), and triple-segment substitution lines (TSSL) for each QTL in the $\mathrm{F}_{3}$ generation using marker-assisted selection (MAS). In addition, analysis of additive and epistatic effects of QTLs on grain length was carried out. The results will be invaluable for the cloning of grain-length and productivity-related genes and their use in rice breeding.

\section{Results}

\section{Identification of substitution segments in Z1392}

Twenty polymorphic SSR markers located in the substitution segments of Z1392, together with 194 polymorphic SSR markers, were used to detect the substitution fragment and assess the homogeneity of the genetic background of Z1392 using 10 individual plants of Z1392. All plants harbored three consistent substitution segments, and no additional chromosomal fragments derived from Xihui 18 were detected. The Z1392 plants were indicated to be homozygous. Three substitution segments of Z1392 originating in Xihui 18 were located on chromosomes 1, 5, and 6. The substitution segment on chromosome 1 was RM3426-RM1167 and its estimated length was $2.40 \mathrm{Mb}$. The substitution segment on chromosome 5 was nSSR505-RM289 and it had an estimated length of $6.97 \mathrm{Mb}$. The substitution segment on chromosome 6 was RM7412-RM494 and its estimated length was $3.50 \mathrm{Mb}$. The total substitution length was $12.87 \mathrm{Mb}$ and the mean length was 4.29 Mb (Fig. 1).

\section{Agronomic traits analysis of Z1392}

Compared with Nipponbare, Z1392 showed a grain length that was increased by $18.43 \%$ and a grain width decreased by $13.49 \%$ ( $p<0.01$ for each trait). Plant height, panicle length, secondary branch number, and number of grains per panicle in Z1392 increased by $33.42 \%, 7.55 \%, 18.45 \%$, and $10.75 \%$ respectively, compared with Nipponbare. The number of panicles in Z1392 was significantly reduced, but was still 10.38 per panicle. No significant differences were observed for other traits between Z1392 and Nipponbare (Fig. 2, Table 1). Taken together, the QTL of these traits may be carried on the substitution fragments of Z1392, which indicates that Z1392 is a valuable genetic resource with potentially important applications in rice breeding.

Table 1 Important agronomic traits for the chromosome segment substitution line Z1392, Nipponbare, and the $\mathrm{F}_{2}$ population 


\begin{tabular}{|c|c|c|c|c|c|c|}
\hline \multirow[t]{2}{*}{ Traits } & \multicolumn{2}{|c|}{ Mean $\pm \mathrm{SD} \square$ Parents $\square$} & \multicolumn{4}{|c|}{$\mathrm{F}_{2}$ population } \\
\hline & Nipponbare & Z1392 & Mean \pm SD & Range & Skew & Kurtosis \\
\hline Plant height (cm) & $89.9 \pm 2.64 * *$ & $119.56 \pm 0.59 * *$ & $106.32 \pm 5.35$ & $93.5-119.4$ & 0.20 & -0.21 \\
\hline Panicle number & $13.20 \pm 3.31 *$ & $10.38 \pm 1.49 *$ & $11.38 \pm 3.09$ & $5-26$ & 0.82 & 1.99 \\
\hline Panicle length $(\mathrm{cm})$ & $20.0 \pm 0.64 * *$ & $21.52 \pm 0.59 * *$ & $21.67 \pm 1.30$ & $18.08-26.22$ & 0.18 & 0.32 \\
\hline Number of primary branches & $8.50 \pm 0.49$ & $8.74 \pm 0.42$ & $8.31 \pm 0.51$ & $7-9.86$ & 0.19 & 0.47 \\
\hline Number of secondary branches & $18.37 \pm 2.27 *$ & $21.76 \pm 2.19 *$ & $29.18 \pm 3.59$ & $19.73-40$ & 0.39 & 0.26 \\
\hline Grain length (mm) & $7 \pm 0.058^{* *}$ & $8.29 \pm 0.068^{* *}$ & $7.80 \pm 0.38$ & 7.1-9.3 & 1.03 & 1.67 \\
\hline Grain width (mm) & $3.4 \pm 0.056^{* *}$ & $2.95 \pm 0.044^{* *}$ & $3.21 \pm 0.11$ & $2.93-3.53$ & 0.14 & 0.16 \\
\hline Grains per panicle & $99.99 \pm 8.97 *$ & $110.74 \pm 7.08 *$ & $102.28 \pm 13.96$ & $61.93-142.8$ & 0.13 & 0.04 \\
\hline 1000-grain weight (g) & $25.44 \pm 0.54$ & $24.73 \pm 0.59$ & $27.72 \pm 7.51$ & $16.29-49.82$ & 0.81 & 0.12 \\
\hline Percentage seed set (\%) & $94.10 \pm 0.90$ & $93.45 \pm 1.41$ & $92.06 \pm 4.02$ & 81.38-99.67 & -0.70 & -0.30 \\
\hline Yield per plant (g) & $31.13 \pm 3.58$ & $28.47 \pm 3.88$ & $27.72 \pm 7.51$ & $16.29-49.82$ & 0.81 & 0.12 \\
\hline
\end{tabular}

Note: * and ** indicate a significant difference between the two parents at $P<0.05$ and $P<0.01$, respectively.

\section{Cytological analysis of glumes in Z1392 and Nipponbare}

To examine the factors responsible for the increase in grain length of the substitution line Z1392, scanning electron microscopy was used to observe the cell morphology of glumes in Nipponbare and Z1392 at the heading stage. We measured cell size in the inner epidermis of the glumes of mature grains. The glume cell length in Z1932 was $25.93 \mu \mathrm{m}$ longer than that of Nipponbare, whereas the cell width was narrower by $4.42 \mu \mathrm{m}$ on average (Fig. 3A, B, D, E, G, H). No significant difference in total cell number in the outer epidermis of the glume along the longitudinal axis was observed between Nipponbare and Z1392 (Fig. 3C, F, I). These findings suggest that the increase in grain length of Z1392 might have resulted predominantly from cell expansion, and not from an increase in cell number.

\section{Genetic analysis of important agronomic traits of Z1392}

The grain shape in Nipponbare displayed a short and broad phenotype, whereas the grain was long and narrow in Xihui 18 and Z1392. The grain shape in $\mathrm{F}_{1}$ individuals derived from the cross between Nipponbare and Z1392 was long and narrow, which indicates that the long grain trait exhibited 
dominance over the short grain phenotype. The skewness and kurtosis of the standard normal distribution were 0 and 3 , respectively. A value of skewness of less than 0 represents a negative skew state, and a value greater than 0 represents a positive skew state. The larger the value, the more severe the skewness and the steeper the kurtosis are. In the $F_{2}$ population of 216 individuals, grain length still showed normal distribution, which indicated that the long-grain phenotype in Z1392 was controlled by at least two dominant genes (Fig. 4A). The other agronomic traits, namely grain width, number of grains per panicle, plant height, panicle length, number of primary branches, number of secondary branches, number of grains per panicle, number of grains per spikelet, 1000-grain weight, and percentage seed set essentially showed normal distributions; skewness was close to zero ( -0.70 to 0.82$)$, which indicated that the skewness was not severe, and the kurtosis ranged from -0.21 to 1.99 (Fig. 4B-K), which indicated that the distribution was uniformly dispersed. Taken together, these results indicated that the agronomic traits were similarly controlled by multiple genes.

\section{QTL mapping for important agronomic traits of Z1392}

18 quantitative trait loci for important agronomic traits were identified in the secondary $F_{2}$ population obtained from the cross between Nipponbare and Z1392. The inheritance of grain length in Z1392 was controlled by two major QTLs, $q G L-5$ and $q G L-6$. The additive effect of alleles of $q G L 5$ and $q G L 6$ inherited from Xihui 18 was estimated to increase the grain length by $0.22 \mathrm{~mm}$ and $0.15 \mathrm{~mm}$ in Z1392, thus the contribution rates were $32.25 \%$ and $15.07 \%$, respectively. The grain width of Z1392 was controlled by the negative effect of $q G W-5$, which could reduce grain width in $Z 1392$ by $0.09 \mathrm{~mm}$ per grain and the contribution rate was $73.38 \%$. The plant height of Z1392 was controlled by four QTLs. Three of these QTLs ( $q P H-1, q P H-5-1$, and $q P H-6)$ showed positive effects and the contribution rates were $7.05 \%, 31.31 \%$, and $31.1 \%$, respectively; $q P H-5-2$ showed a negative effect with a contribution rate of $22.53 \%$. Three QTLs controlled panicle length, of which $q P L-5-1$ and $q P L-6$ showed positive effects, and the contribution rates were $11.79 \%$ and $20.26 \%$, respectively; $q P L-5-2$ showed a negative effect with a contribution rate of 13.44\%. The QTL $q P N-1$, located on chromosome 1, showed a negative effect on the number of effective panicles with a contribution rate of $3.14 \%$. The QTL $q G P-1$ showed an additive effect on the number of grains per panicle of Z1392, which was increased by 4.52 , and the contribution rate was $5.02 \%$. Four QTLs for percentage seed set were detected in the substitution segments of chromosomes 1, 5, and 6 . Three of these QTLs ( $q S S R-1, q S S R-5-2$, and $q S S R-6$ ) showed positive effects and the contribution rates were $2.84 \%, 24.80 \%$, and $49.62 \%$, respectively; $q S S R-5-1$ showed a negative effect with a contribution rate of 49.30\%. TWo QTLs ( $q K W T-5$ and $q K W T-6$ ) showed negative and positive effects on 1000-grain weight, and the contribution rates were $20.12 \%$ and $21.81 \%$, respectively (Table 2 ).

Table 2 QTLs associated with important agronomic traits detected in the chromosome substitution segments of Z1392 


\begin{tabular}{|c|c|c|c|c|c|c|}
\hline Traits & QTL & Chr. & Linked marker & $\begin{array}{l}\text { Estimated } \\
\text { effect } \pm S E\end{array}$ & Var\% & P-value \\
\hline Plant height & $q P H-1$ & 1 & RM3426 & $2.11 \pm 0.76$ & 7.05 & 0.0058 \\
\hline Plant height & $q P H-5-1$ & 5 & RM18119 & $4.44 \pm 1.46$ & 31.31 & 0.0026 \\
\hline Plant height & $q P H-5-2$ & 5 & nSSR505 & $-3.77 \pm 1.12$ & 22.53 & 0.0009 \\
\hline Plant height & $q P H-6$ & 6 & RM7412 & $4.43 \pm 1.45$ & 31.10 & 0.0025 \\
\hline Panicle length & $q P L-5-1$ & 5 & RM18119 & $0.62 \pm 0.31$ & 11.79 & 0.0494 \\
\hline Panicle length & $q P L-5-2$ & 5 & nSSR505 & $-0.66 \pm 0.25$ & 13.44 & 0.0089 \\
\hline Panicle length & $q P L-6$ & 6 & RM7412 & $0.81 \pm 0.31$ & 20.26 & 0.0093 \\
\hline Panicle number & $q P N-1$ & 1 & RM3426 & $-0.55 \pm 0.27$ & 3.14 & 0.0397 \\
\hline Grain length & $q G L-5$ & 5 & RM18119 & $0.22 \pm 0.07$ & 32.25 & 0.0012 \\
\hline Grain length & $q G L-6$ & 6 & RM7412 & $0.15 \pm 0.07$ & 15.07 & 0.0282 \\
\hline Grain width & $q G W-5$ & 5 & nSSR505 & $-0.09 \pm 0.02$ & 73.38 & $<0.0001$ \\
\hline Grains per panicle & $q G P-1$ & 1 & RM3426 & $4.52 \pm 1.79$ & 5.02 & 0.0124 \\
\hline percentage seed set & $q S S R-1$ & 1 & RM3426 & $1.97 \pm 0.97$ & 2.84 & 0.0423 \\
\hline percentage seed set & $q S S R-5-1$ & 5 & RM18119 & $-7.35 \pm 1.92$ & 49.30 & 0.0002 \\
\hline percentage seed set & $q S S R-5-2$ & 5 & nSSR505 & $5.21 \pm 1.45$ & 24.80 & 0.0004 \\
\hline percentage seed set & $q S S R-6$ & 6 & RM494 & $7.37 \pm 1.96$ & 49.62 & 0.0002 \\
\hline 1000-grain weight & $q K W T-5$ & 5 & nSSR505 & $-1.08 \pm 0.33$ & 20.12 & 0.0015 \\
\hline 1000-grain weight & $q K W T-6$ & 6 & RM7412 & $1.12 \pm 0.42$ & 21.81 & 0.0075 \\
\hline
\end{tabular}

\section{Fine mapping of putative $q G L 6$ and sequence analysis of candidate genes}

On the basis of QTL mapping, 241 recessive individuals with short grains excluded them with bands of Nipponbare in $q G L-5$ locus of the $F_{2}$ population was used to fine map $q G L-6$. Finally, $q G L-6$ was fine mapped between RM439 and RM103 on the chromosome 6, with the physical distance of $1.26 \mathrm{Mb}$.

Through gene prediction and sequencing, the auxin response factor OsARF19 was identified as a candidate gene of $q G L-6$, and there were many differences in the DNA sequence of OsARF19 in Nipponbare and Z1392. Firstly, there are 6 CAGs in the CAG repeat region after $1803^{\text {rd }}$ bases in Nipponbare, and only 5 CAGs in Z1392, and the CAG encodes glutamine. Thus, a glutamine of the OSARF19 protein in Z1392 is reduced compared to Nipponbare. Secondly, a base in the $1830^{\text {th }}$ and $1833^{\text {rd }}$ bases in Nipponbare was changed to $\mathrm{G}$ base in Z1392. Thus the CAA in Nipponbare was changed to the CAG in Z1392, but both of them encoded glutamine, and did not cause amino acid changes. 


\section{Analysis of additive and epistatic effects of QTLs on grain length}

On the basis of the QTL mapping results, eight individuals were selected from the $F_{2}$ population by MAS, and $F_{3}$ lines were obtained from each individual. Ten individuals for each $F_{3}$ line were sampled for further molecular marker selection using heterozygous markers in the selected plant lines. Ultimately, three SSSLs (S1, S2, and S3) and two DSSLs (D1 and D2) were selected. S1 carried the substitution fragment of chromosome 1 and lacked the QTLs for grain length and grain width. S2 carried the substitution fragment of chromosome 5, which included the grain length QTL $q G L-5$, for which the additive effect was estimated to be an increased grain length of $S 2$ by $0.3 \mathrm{~mm}$. S3 carried the chromosome 6 substitution fragment containing the grain length QTL $q G L-6$, for which the additive effect was estimated to be an increased grain length of S3 by $0.13 \mathrm{~mm}$ (Fig. 5). These QTLs were repeatedly detected in different years ( $q G L-5: \mathrm{a}_{2017}=0.22, \mathrm{P}=0.0012 ; \mathrm{a}_{2018}=0.22, \mathrm{P}=0.0012 ; q G L-6: \mathrm{a}_{2017}=0.15, \mathrm{P}=0.028 ; \mathrm{a}_{2018}=0.22, \mathrm{P}=0.0012$ ), which indicate that the QTLs were mapped accurately, and differences in the additive effect of the QTLS were detected in comparison with the previous season, which suggests that different environments have interactive effects on grain length.

The DSSL D1 carried the substitution fragments of chromosomes 1 and 6 . The substitution fragment of chromosome 1 lacked a grain length QTL, whereas the substitution fragment of chromosome 6 contained $q G L-6$. The additive effect of $q G L-6$ was $0.13 \mathrm{~mm}$, and an epistatic effect between the two substitution segments of $0.41 \mathrm{~mm}$ was observed. The DSSL D2 carried substitution fragments of chromosomes 5 and 6 . The substitution fragment of chromosome 5 contained $q G L-5$, for which the additive effect was $0.3 \mathrm{~mm}$, the substitution fragment of chromosome 6 contained $q G L-6$, which showed an additive effect of $0.13 \mathrm{~mm}$, and an epistatic effect between the two substitution fragments of 0.31 mm was observed. The TSSL T3 (Z1392) carried the three substitution fragments. As indicated in the preceding discussion, the substitution fragment of chromosome 1 lacked a grain length QTL, whereas those of chromosomes 5 and 6 contained $q G L-5$ and $q G L-6$, respectively. An epistatic effect among the three substitution fragments of $0.26 \mathrm{~mm}$ was observed. Therefore, the theoretical genetic effect on grain length of D1, D2, and T3 was $0.44 \mathrm{~mm}, 0.74 \mathrm{~mm}$, and $0.69 \mathrm{~mm}$, respectively. Given that the grain length of the recipient parent Nipponbare was $7.2 \mathrm{~mm}$, the grain length of D1, D2, and T3 was predicted to be $8.15 \mathrm{~mm}, 8.29 \mathrm{~mm}$, and $8.24 \mathrm{~mm}$, respectively. However, the actual average grain length of D1, D2, and T3 was $8.45 \mathrm{~mm}, 8.67 \mathrm{~mm}$, and $8.58 \mathrm{~mm}$, which may be the result of error or environmental effects between individuals (Fig. 5). Taken together, the combination of $q G L-5$ and $q G L-6$ resulted in development of a longer grain.

\section{Discussion}

In this study, the rice CSSL Z1392 was identified, having been derived from a cross between Nipponbare as the recipient parent and the indica restorer line Xihui 18 as the donor parent. Z1392 carried three substitution segments and exhibited a long-grain phenotype. Z1392 exhibited many phenotypic traits beneficial for rice breeding and thus represents an important genetic resource. Traits such as plant length, grain length, panicle length, number of secondary branches per panicle, and filled grain number per 
panicle were significantly higher in Z1392 than in Nipponbare. The major QTLs carried by Z1392, qGL-5 and $q G L-6$, contributed to grain length, which thus affords the opportunity for future research on the molecular mechanisms underlying the development and regulation of grain length. In addition, we analyzed two SSSLs that carried $q G L-5$ or $q G L-6$, which positively affected grain length, and one DSSL that carried $q G L-5$ and $q G L-6$. Interaction of $q G L-5$ and $q G L-6$ resulted in positive epistatic effects in the DSSL, which increased the grain length compared with the single long-grain SSSL. Thus, combination of major QTLs that positively affect grain length resulted in a further increase in grain length. However, a previous study showed that combination of two QTLs, qGL3 and $q G L 4-b$, which each have positive effects on grain length, resulted in negative epistatic effects in the DSSL and thus did not result in a longer grain (Zhao et al., 2011). Nevertheless, these results are not contradictory, but rather verify the finding that QTLs with different effects on grain length will produce different interactive effects when combined, and only when the epistatic effect is in the same direction as the additive effect of the target gene can any significant improvement in grain length be achieved. To achieve the desired effect of the target gene, it is first necessary to predict whether two genes interact with each other and the effect of that interaction. Therefore, given that $Z 1392$ harbors $q G L-5$ and $q G L-6$, the line is an important resource for molecular breeding of rice.

Eighteen QTLs for agronomic traits were identified in Z1392. Among these QTLs, four control plant height: $q P H-1$ is located on chromosome 1 in the same region as the dwarfism gene $D 18$, which is a gene involved in gibberellin synthesis and affects plant height development by regulating BR and phytochrome (Tong et al, 2014); $q P H-6$ is located in the same chromosomal region as OsLIC1, which belongs to the BR regulatory pathway, and regulates the development of plant height, leaf angle size, and grain number per panicle (Wang et al, 2008); $q P H-5-1$ and $q P H-5-2$ are QTLs associated with plant height located in the same substitution fragment, which also contains the OSBLE3. OSBLE3 is specifically up-regulated by BR and is involved in cell elongation under dual regulation by brassinolide and indoleacetic acid (Yang et al, 2006). Given that these genes regulate the process of hormone synthesis or signal transcription, we speculated that the plant height of Z1392 may also be regulated by hormones; therefore combination of the above genes could be used to breed rice cultivars of ideal height. The percentage seed-set QTL $q S S R$ 6 carried by Z1392 was mapped to the same locus as OSARF18, which regulates the auxin signaling pathway (Huang et al, 2016); qSSR-5-1 and qSSR-5-2 were mapped to the same locus as OSCYP51G3, for which RNA interference causes dwarfism, decreased percentage seed set, shortened stem cells, and decreased leaf inclination (Xia et al, 2015). The grain size QTLs $q G W-5$ and $q K W T-5$ are located in the same chromosomal region as GW5, which participates in the ubiquitin-proteasome pathway to regulate cell division during seed development (Weng et al, 2008). The grain length QTL $q G L-6$ was fine mapped on the chromosome 6, with the physical distance of $1.26 \mathrm{Mb}$. OsARF19 may be the candidate gene of it, which encodes an auxin response factor, mutation of it displayed enlarged organs caused by cell elongation(Zhang et al, 2015). The localization interval for the grain length QTL $q G L-5$ may contain five genes associated with the development of grain size and which encode a Serine/Threonine protein phosphatase, a rapid alkalization factor (RALF) family protein precursor, a GSK3/SHAGGY-Like kinase, a protein kinase, and an expressed protein containing a PPR repeat sequence. Although these genes that 
control different traits are located in the same chromosomal interval as the QTLs identified in the present study, further sequencing and functional complementation are needed to determine whether the genes are alleles of the identified QTLs. In addition, $q P L-5-1, q P L-5-2, q P L-6, q P N-1, q G P-1$, and $q S S R-1$ have not been reported previously. Therefore, the present study lays the foundations for additional fine localization of the QTLs, cloning of the candidate genes, and functional research on grain size.

\section{Conclusions}

The rice TSSL line Z1392 was identified, which exhibited increased grain length. The chromosomal substitution fragments were located on chromosomes 1,5 , and 6 , and the average substitution length was $4.29 \mathrm{Mb}$. The increased grain length in Z1392 might be caused predominantly by cell expansion in the glumes. Inheritance of the long-grain phenotype in Z1392 is controlled by two major QTLs, $q G L-5$ and qGL-6. $q G L-6$ was narrowed to a $1.26 \mathrm{Mb}$ region on chromosome 6, and OSARF19 may be the candidate gene of $q G L-6$. On the basis of QTL mapping, three SSSLs and two DSSLs were selected. Epistatic effects analysis revealed a positive epistatic interaction between $q G L-5$ and $q G L-6$, which indicates that pyramiding of $q G L-5$ and $q G L-6$ enhances the long-grain phenotype.

\section{Materials And Methods}

\section{Plant materials}

The rice CSSL Z1392 was developed using the rice cultivars 'Nipponbare' as the recipient parent and 'Xihui 18 ' as the donor parent. After repeated backcrossing and selfing, in combination with phenotypebased selection and simple sequence repeat (SSR) marker selection, a genetically stable CSSL with three substitution segments was identified and designated Z1392.

The plant material used for QTL mapping was a secondary $F_{2}$ population derived from a cross between Nipponbare and Z1392.

On the basis of QTL mapping results obtained in 2017, eight individual plants were selected by MAS and planted in 2018. Ten individual plants were selected from each line to allow further selection of molecular markers for hybrid markers, and homozygous single-fragment substitution lines and double-fragment substitution lines were then selected.

\section{Material planting method}

In June 2016, the $F_{1}$ was generated at the experimental station of Southwest University in Chongqing, China, by crossing Nipponbare with Z1392. In August, hybrid seeds were planted in LingShui, Hainan Province. All $30 \mathrm{~F}_{1}$ seeds and seeds of the parents were planted at the experimental station of Southwest University in Chongqing on March 10, 2017. In March 2018, on the basis of QTL mapping results in 2017, 30 seeds of eight individual plants were used for breeding of secondary substitution fragment lines, and the parents were planted in the same experimental field. On April 15, 2018, all plants were transplanted to 
the same experimental field. The spacing between hills was $16.67 \mathrm{~cm}$ and the spacing between rows was $26.67 \mathrm{~cm}$. Conventional field management practices were applied.

\section{Identification of substitution segments in Z1392}

A set of 263 markers polymorphic between Nipponbare and Xihui 18 were selected from 429 markers that covered the entire rice genome. The long-grain substitutions line Z1392, harboring three substitution segments, was selected from the $\mathrm{BC}_{3} \mathrm{~F}_{4}$ generation by selection of molecular marker and phenotype. The identification of substitution segments was performed as described previously (Zhao et al, 2016), and the estimated length of the substitution segments was calculated following an established method (Paterson et al, 1991). The distance of the substitution markers from the donor plus half of the distance between the boundary markers from Nipponbare and the substitution markers was regarded to be the estimated substitution length. Mapchart 2.2 was used to draw a chromosome substitution fragment map.

\section{Agronomic trait assessment}

At maturity, 10 plants on the third to seventh hills of the central two rows of the Nipponbare and Z1392 plots, and 10 plants of selected SSSLs and DSSLs, and 216 individuals for QTL mapping were harvested. For each plant the grain length, grain width, grain number per panicle, filled grain number per panicle, percentage seed set, plant height, number of primary branches per panicle, number of secondary branches per panicle, number of effective panicles, grain weight per plant, panicle length, and 1000-grain weight were measured. A Student's t-test for each trait to assess the significance of differences between Nipponbare and Z1392, and descriptive statistics, such as skewness and kurtosis, in the $F_{2}$ population was conducted using the statistical functions implemented in Microsoft Excel 2010.

\section{Scanning electron microscopy}

At the completion of the booting stage and before the heading period, the phenotypic characteristics of the inner and outer epidermal cells of the glume in Nipponbare and Z1392 were investigated using a Hitachi SU3500 scanning electron microscope (Hitachi, Tokyo, Japan) with a frozen stage $\left(-40^{\circ} \mathrm{C}\right)$ under a low-vacuum environment.

\section{Genetic analysis of important agronomic traits of Z1392}

The Excel 2010 statistical function was used to calculate the average, minimum, maximum, skewness, and kurtosis of the aforementioned 8 agronomic traits of the 216 individual plants used for QTL mapping in the $F_{2}$ population. A histogram of the frequency distribution for each trait was generated to assess their genetic characteristics.

\section{QTL mapping}

Total genomic DNA of Nipponbare, Xihui 18, Z1392, and the 216 plants from the $F_{2}$ population was extracted using the cetyltrimethylammonium bromide method (Mccouch et al, 1988). PCR amplification, 
non-denaturing polyacrylamide gel electrophoresis, and rapid silver staining were performed as described previously (Zhao et al, 2016). Nipponbare bands were scored as "-1", Z1392 bands were scored as "1", heterozygous bands were scored as " 0 ", and the absence of marker bands was scored as ".." The marker assignments of all six SSR markers on the substitution segments of Z1392, together with the phenotypic values of each individual in the $F_{2}$ population, were used for QTL mapping. QTL mapping was performed using the restricted maximum likelihood method by mixed linear models(MLM) implemented in the HPMIXED procedure of SAS software (SAS Institute Inc., Cary, NC, USA), with significance determined at $a=0.05$ (Hu and Xu, 2009, Cui et al, 2017, Spilke et al, 2005).

\section{Development of SSSLS and DSSLs, and additive and epistatic effects analysis}

On the basis of the QTL mapping results in 2017, eight plants were selected using a MAS method and were planted in 2018. Ten plants from each line were selected to select further molecular marker assisted selection. Ultimately, homozygous SSSLs and DSSLs were selected. At the maturity stage, 10 plants from each SSSL, DSSL, and TSSL (Z1392) were sampled and the grain length and grain width of each plant were measured, with three replicate measurements per plant recorded. Student's $t$-tests for each substitution line and descriptive statistics, such as average and standard deviation, were conducted using the statistical functions implemented in Excel 2010. Additive and epistatic effects analysis was performed as described previously (Eshed and Zamir, 1996).

\section{List Of Abbreviations}

QTL: Quantitative trait loci; SSSL: single-segment; CSSLs: Chromosome segment substitution lines; DSSL: double-segment; TSSL: triple-segment substitution lines; SSR: Simple sequence repeat; SEM: Scanning electron microscopy; MAS: marker-assisted selection.

\section{Declarations}

\section{Ethics approval and consent to participate}

This study complied with the ethical standards of China, where this research work was carried out.

\section{Consent for publication}

All authors are consent for publication.

\section{Availability of data and materials}

The datasets supporting the conclusions of this article are included within the article.

\section{Competing interests}

The authors declare that they have no competing interests. 


\section{Funding}

The study was supported by the National Key Research Plan Project (2017YFD0101107), the National Natural Science Foundation of China (grant no. 31900612), and the Chongqing Technical Innovation and Application Development Project (cstc2019jscx-msxmX0392).

\section{Authors' contributions}

TZ, SMW and FMZ conceived and designed the experiments. TZ, SMW, SGS, YZ, JL, JY, TS and WBC performed the experiments. TZ, SMW, YHL, GHH and FMZ analyzed the data. TZ and FMZ wrote the paper. All authors read and approved the final manuscript.

\section{Acknowledgments}

We thank Professor Shizhong Xu at University of California, Riverside, USA for writing the stem program for the QTL mapping.

\section{References}

Bai X. F., Luo L. J., Yan W. H., Kovi M. R., Zhan W., Xing Y. Z. 2010. Genetic dissection of rice grain shape using a recombinant inbred line population derived from two contrasting parents and fine mapping a pleiotropic quantitative trait locus qGL7. Bmc Genetics, 11.

Cui T. T., He K. H., Chang L. G., Zhang X. H., Xue J. Q., Liu J. C. 2017. QTL mapping for leaf area in maize (Zea mays L.) under multi-environments. Journal Of Integrative Agriculture, 16(4): 800-808.

Duan P. G., Rao Y. C., Zeng D. L., Yang Y. L., Xu R., Zhang B. L., Dong G. J., Qian Q., Li Y. H. 2014. SMALL GRAIN 1, which encodes a mitogen-activated protein kinase kinase 4 , influences grain size in rice. Plant Journal, 77(4): 547-557.

Eshed Y., Zamir D. 1996. Less-than-additive epistatic interactions of quantitative trait loci in tomato. Genetics, 143(4): 1807-1817.

Fang N., Xu R., Huang L. J., Zhang B. L., Duan P. G., Li N., Luo Y. H., Li Y. H. 2016. SMALL GRAIN 11 Controls Grain Size, Grain Number and Grain Yield in Rice. Rice, 9.

Guo T., Chen K., Dong N. Q., Shi C. L., Ye W. W., Gao J. P., Shan J. X., Lin H. X. 2018. GRAIN SIZE AND NUMBER1 Negatively Regulates the OsMKKK10-OsMKK4-OsMPK6 Cascade to Coordinate the Trade-off between Grain Number per Panicle and Grain Size in Rice. Plant Cell, 30(4): 871-888.

Hu Z., Xu S. 2009. PROC QTL-A SAS Procedure for Mapping Quantitative Trait Loci. Int J Plant Genomics, 2009: 141234. 
Huang J., Li Z., Zhao D. 2016. Deregulation of the OsmiR160 Target Gene OsARF18 Causes Growth and Developmental Defects with an Alteration of Auxin Signaling in Rice. Sci Rep, 6: 29938.

Jin J., Hua L., Zhu Z. F., Tan L. B., Zhao X. H., Zhang W. F., Liu F. X., Fu Y. C., Cai H. W., Sun X. Y., Gu P., Xie D. X., Sun C. Q. 2016. GAD1 Encodes a Secreted Peptide That Regulates Grain Number, Grain Length, and Awn Development in Rice Domestication. Plant Cell, 28(10): 2453-2463.

Kubo T., Yoshimura A. 2002. Genetic basis of hybrid breakdown in a Japonica/Indica cross of rice, Oryza sativa L. Theoretical And Applied Genetics, 105(6-7): 906-911.

Li N., Li Y. H. 2016. Signaling pathways of seed size control in plants. Current Opinion in Plant Biology, 33: 23-32.

Liu L. C., Tong H. N., Xiao Y. H., Che R. H., Xu F., Hu B., Liang C. Z., Chu J. F., Li J. Y., Chu C. C. $2015 a$. Activation of Big Grain1 significantly improves grain size by regulating auxin transport in rice (vol 112, pg 11102, 2015). Proc Natl Acad Sci U S A, 112(39): E5446-E5446.

Liu Q., Han R. X., Wu K., Zhang J. Q., Ye Y. F., Wang S. S., Chen J. F., Pan Y. J., Li Q., Xu X. P., Zhou J. W., Tao D. Y., Wu Y. J., Fu X. D. 2018. G-protein beta gamma subunits determine grain size through interaction with MADS-domain transcription factors in rice. Nat Commun, 9.

Liu S. Y., Hua L., Dong S. J., Chen H. Q., Zhu X. D., Jiang J. E., Zhang F., Li Y. H., Fang X. H., Chen F. 2015 b. OsMAPK6, a mitogen-activated protein kinase, influences rice grain size and biomass production. Plant Journal, 84(4): 672-681.

Mccouch S. R., Kochert G., Yu Z. H., Wang Z. Y., Khush G. S., Coffman W. R., Tanksley S. D. 1988. Molecular Mapping Of Rice Chromosomes. Theoretical And Applied Genetics, 76(6): 815-829.

Paterson A. H., Damon S., Hewitt J. D., Zamir D., Rabinowitch H. D., Lincoln S. E., Lander E. S., Tanksley S. D. 1991. Mendelian Factors Underlying Quantitative Traits In Tomato - Comparison across Species, Generations, And Environments. Genetics, 127(1): 181-197.

Song X. J., Huang W., Shi M., Zhu M. Z., Lin H. X. 2007. A QTL for rice grain width and weight encodes a previously unknown RING-type E3 ubiquitin ligase. Nat Genet, 39(5): 623-630.

Song X. J., Kuroha T., Ayano M., Furuta T., Nagai K., Komeda N., Segami S., Miura K., Ogawa D., Kamura T., Suzuki T., Higashiyama T., Yamasaki M., Mori H., Inukai Y., Wu J. Z., Kitano H., Sakakibara H., Jacobsen S. E., Ashikari M. 2015. Rare allele of a previously unidentified histone $\mathrm{H} 4$ acetyltransferase enhances grain weight, yield, and plant biomass in rice. Proc Natl Acad Sci U S A, 112(1): 76-81.

Spilke J., Piepho H. P., Hu X. 2005. Analysis of unbalanced data by mixed linear models using the MIXED procedure of the SAS system. Journal of Agronomy and Crop Science, 191(1): 47-54. 
Tong H., Xiao Y., Liu D., Gao S., Liu L., Yin Y., Jin Y., Qian Q., Chu C. 2014. Brassinosteroid regulates cell elongation by modulating gibberellin metabolism in rice. Plant Cell, 26(11): 4376-4393.

Wang L., Xu Y., Zhang C., Ma Q., Joo S. H., Kim S. K., Xu Z., Chong K. 2008. OsLIC, a Novel CCCH-Type Zinc Finger Protein with Transcription Activation, Mediates Rice Architecture via Brassinosteroids Signaling. PLoS One, 3(10): e3521.

Weng J. F., Gu S. H., Wan X. Y., Gao H., Guo T., Su N., Lei C. L., Zhang X., Cheng Z. J., Guo X. P., Wang J. L., Jiang L., Zhai H. Q., Wan J. M. 2008. Isolation and initial characterization of GW5, a major QTL associated with rice grain width and weight. Cell Res, 18(12): 1199-1209.

Xia K. F., Ou X. J., Tang H. D., Wang R., Wu P., Jia Y. X., Wei X. Y., Xu X. L., Kang S. H., Kim S. K., Zhang M. Y. 2015. Rice microRNA osa-miR1848 targets the obtusifoliol 14 alpha-demethylase gene OsCYP51G3 and mediates the biosynthesis of phytosterols and brassinosteroids during development and in response to stress. New Phytologist, 208(3): 790-802.

Yang G. X., Nakamura H., Ichikawa H., Kitano H., Komatsu S. 2006. OsBLE3, a brassinolide-enhanced gene, is involved in the growth of rice. Phytochemistry, 67(14): 1442-1454.

Zhang S, Wu T , Liu S, et al. Disruption of OsARF19 is Critical for Floral Organ Development and Plant Architecture in Rice (Oryza sativa L.)[J]. Plant Molecular Biology Reporter, 2015, 34(4).

Zhang X. Q., Sun J., Cao X. F., Song X. W. 2015. Epigenetic Mutation of RAV6 Affects Leaf Angle and Seed Size in Rice. Plant Physiol, 169(3): 2118-2128.

Zhao F. M., Tan Y., Zheng L. Y., Zhou K., He G. H., Ling Y. H., Zhang L. H., Xu S. Z. 2016. Identification of Rice Chromosome Segment Substitution Line Z322-1-10 and Mapping QTLs for Agronomic Traits from the F-3 Population. Cereal Research Communications, 44(3): 370-380.

Zhao F. M., Zhang G. Q, Zeng R. Z, Yang Z. L, Ling Y. H, Sang X. C, He G. H. 2011. Analysis of Epistatic and Additive Effects of QTLs for Grain Shape Using Single Segment Substitution Lines in Rice (Oryza sativa L.). ACTA AGRONOMICA SINICA (Chinese), 201 1, 37(3): 469-476.

\section{Figures}



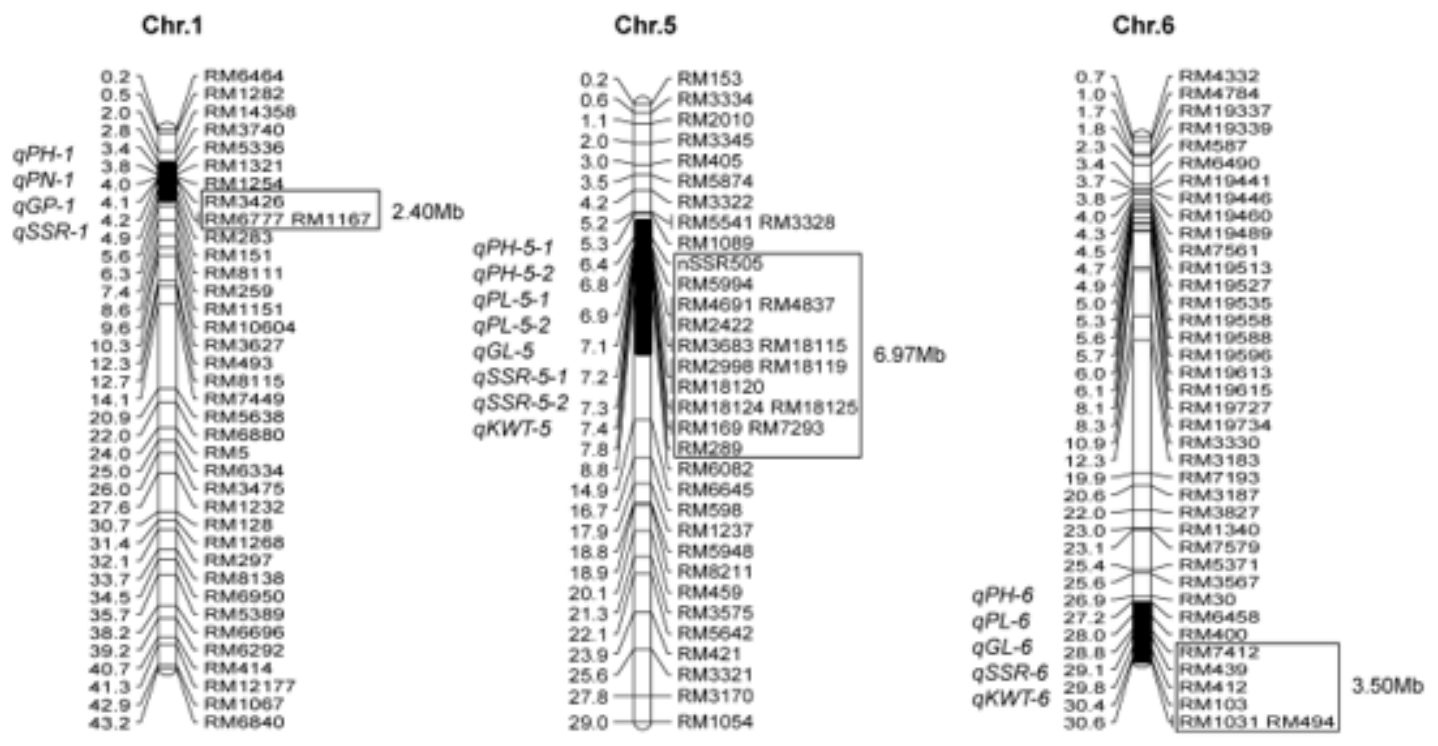

\section{Figure 1}

Substitution segments of Z1392. Physical distance $(\mathrm{Mb})$ is specified on the left of each chromosome (based on the Nipponbare reference genome) and markers are specified on the right. The solid black segment is the substitution fragment region from the donor Xihui 18 and the identified QTLs are listed on the left of each chromosome in italics. The estimated length of the chromosomal substitution fragment is marked on the right of each chromosome beside the box. The markers enclosed in each box include the boundary markers and the substitution markers. qPH, QTL for plant height; qPN, QTL for panicle number; qGP, QTL for grains per panicle; qSSR, QTL for percentage seed set; qPL, QTL for panicle length; qGL, QTL for grain length; qKWT, QTL for 1000-grain weight.

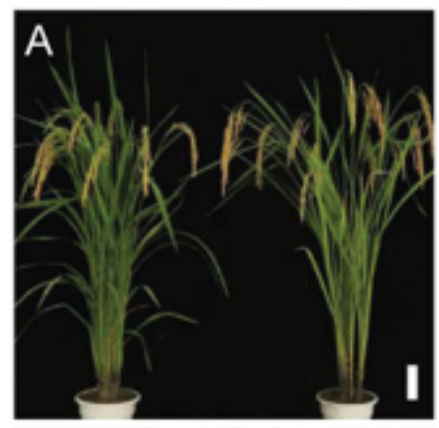

C
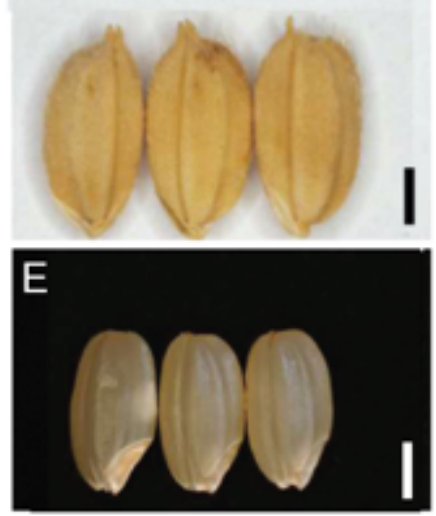
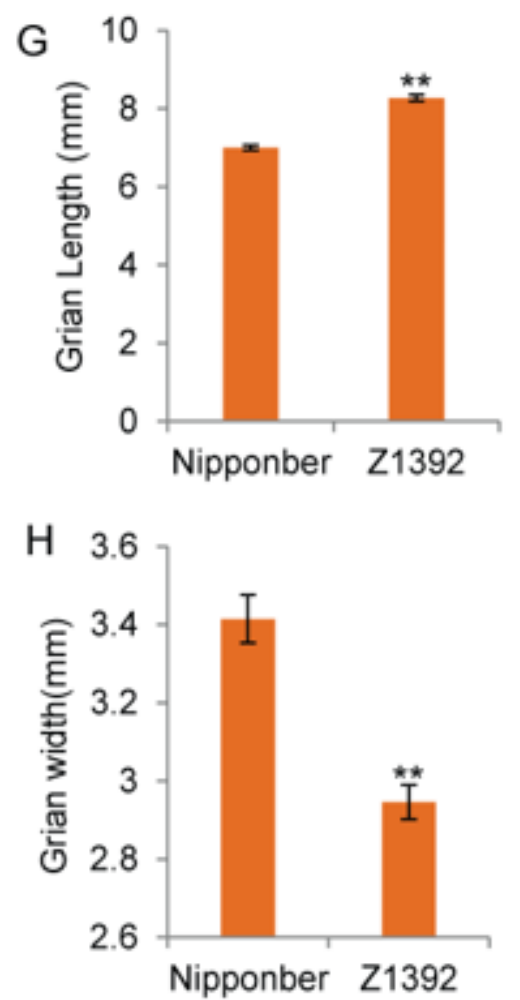


\section{Figure 2}

Phenotype of Nipponbare and Z1392. A, Plant type of Nipponbare (left) and Z1392 (right). B, Terminal panicle of Nipponbare (left) and Z1392 (right). C, D, Grains of Nipponbare (C) and Z1392 (D). E, F, Brown grains of Nipponbare (E) and Z1392 (F). G, H, Grain length and grain width of Nipponbare and Z1392. Bars in $A$ and $B, 10 \mathrm{~cm}$; in $C-F, 2 \mathrm{~mm}$.

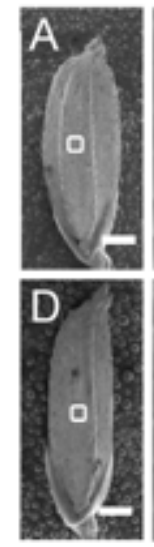

G
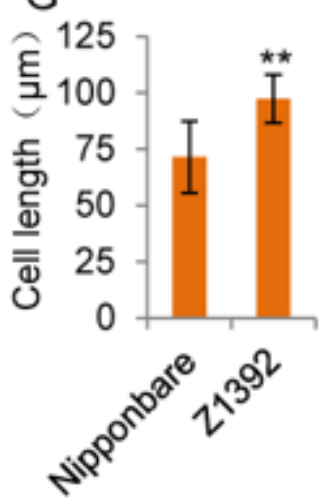
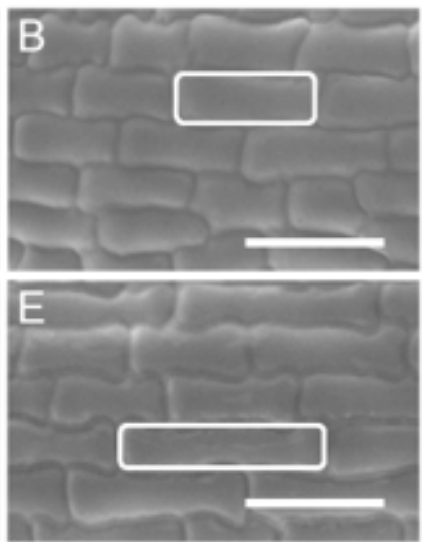

$\mathrm{H}$
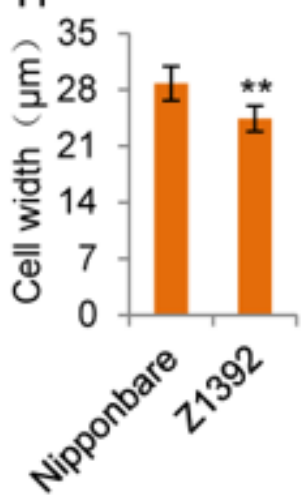
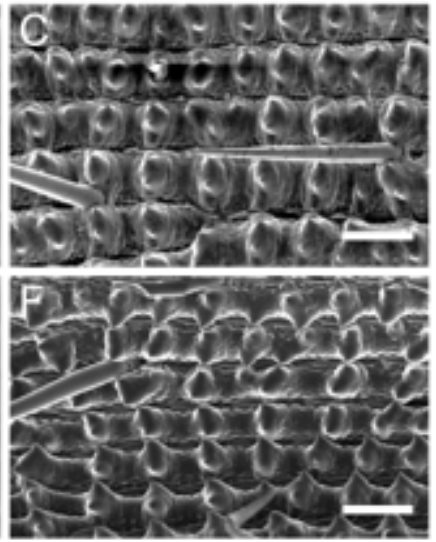

I
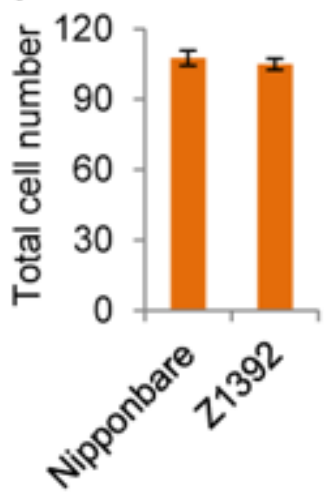

Figure 3

Scanning electron microscopic observation and analysis of the glume. A-C, Scanning electron micrograph of the lemma $(A)$, and inner epidermis $(B)$ and outer epidermis $(C)$ of the glume of Nipponbare. D-F, Scanning electron micrograph of the lemma (D), and inner epidermis (E) and outer epidermis (F) of the glume of Z1392. G, Cell length in the inner epidermis of the lemma of Nipponbare and Z1392. H, Cell width in the inner epidermis of the lemma of Nipponbare and Z1392. I, Total cell number in the outer epidermis of the lemma along the longitudinal axis of Nipponbare and Z1392. Bars in $A$ and $B, 1 \mathrm{~mm} ; B, C, E$ and $F, 100 \mu \mathrm{m}$. * and ** indicate a significant difference between the two parents at $P<0.05$ and $P<0.01$, respectively. 


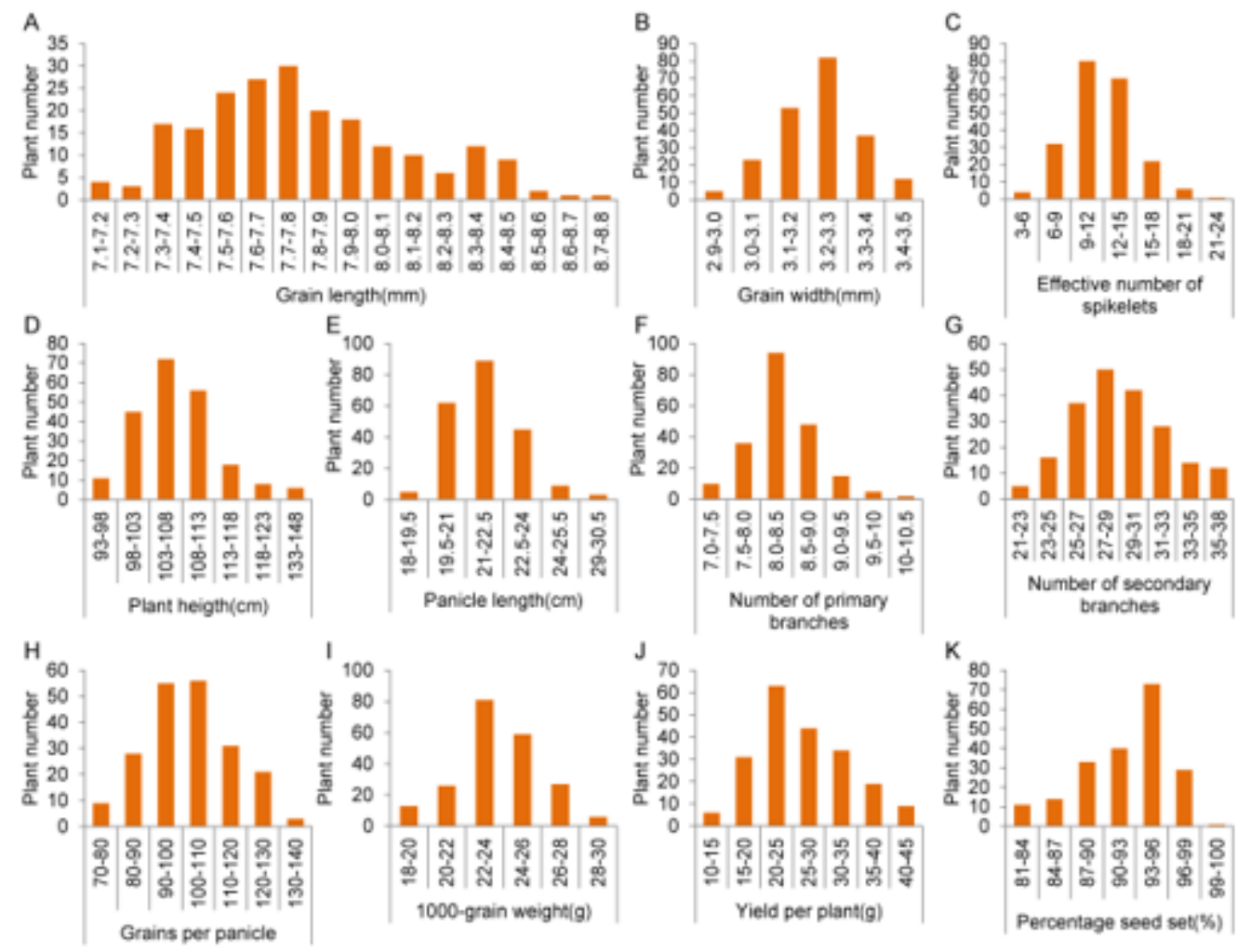

Figure 4

Frequency distributions of agronomic traits in the F2 population. A, Grain length; B, grain width; C, panicle number; $D$, plant height; $E$, panicle length; $F$, number of primary branches; $G$, number of secondary branches; $\mathrm{H}$, number of grains per panicle; I, 1000-grain weight; $J$, yield per plant; $\mathrm{K}$, seed set.

Chr 6
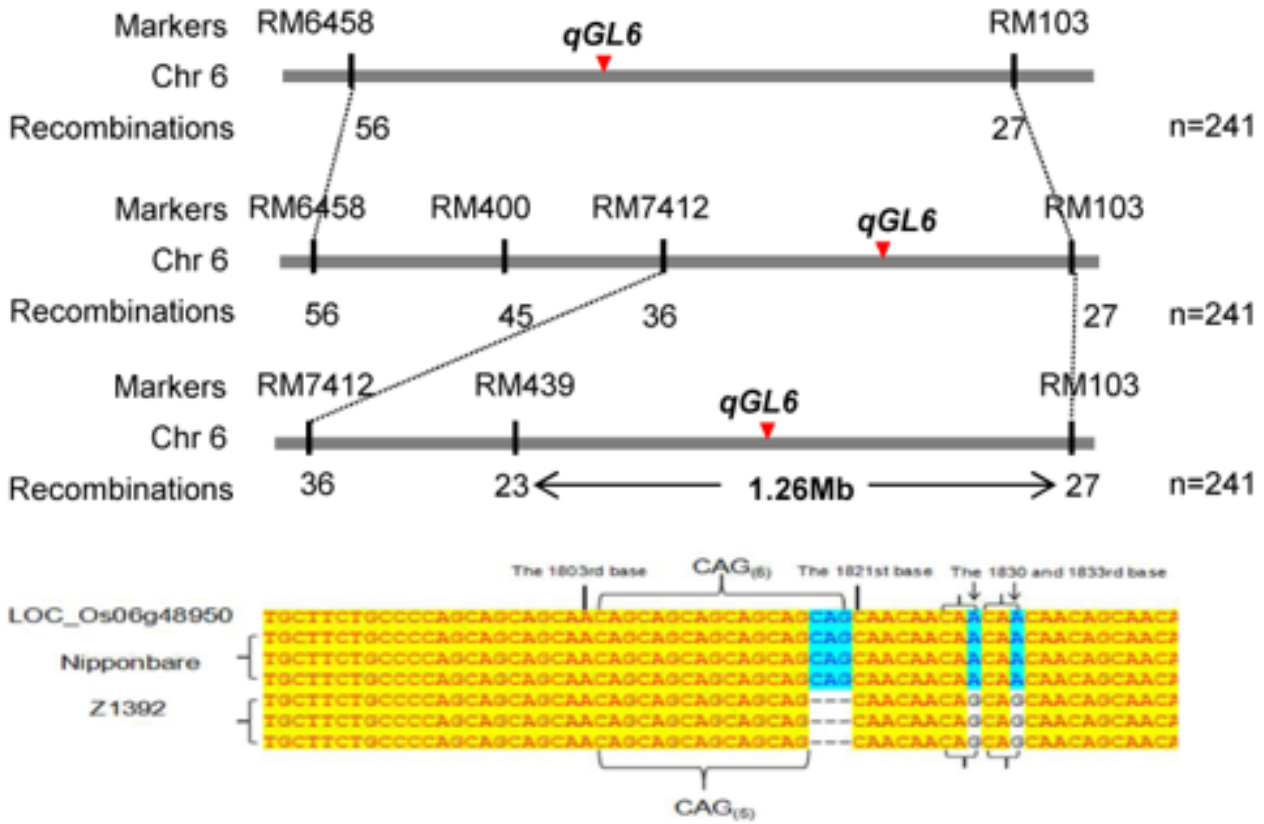

Figure 5 
Fine mapping of putative qGL6 and sequence analysis of candidate genes.

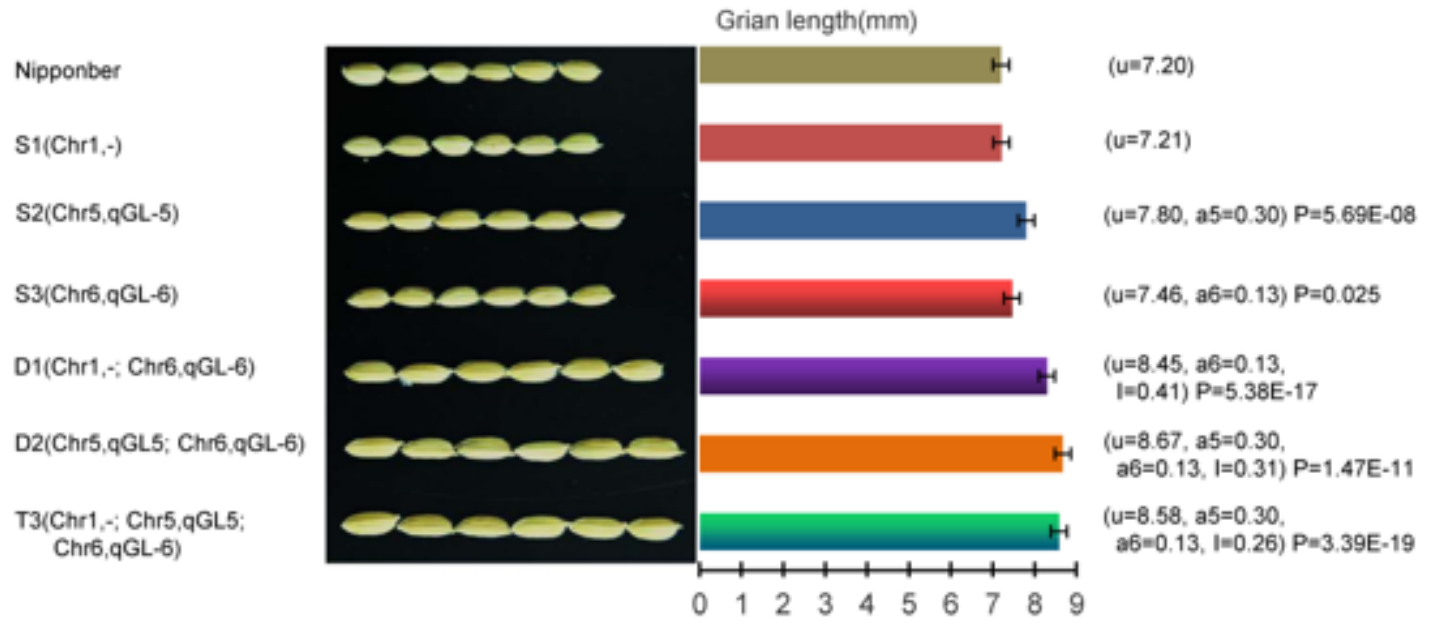

\section{Figure 6}

Phenotype and genotype analysis of six chromosome segment substitution lines. $u$ is the phenotypic value; a denotes the additive effect; I denotes the epistatic effect. t-test was used to significant difference analysis. 